\title{
UTILITY AND IMPLEMENTATION OF REFLECTION IN DISTINCT TYPES OF ENGINEERING COURSES
}

\author{
Ibrahim El Bojairami ${ }^{1}$, Sonya Kouthouridis ${ }^{2}$, Zeinab Sobhanigavgani ${ }^{3}$, Maxime Jacques ${ }^{3}$, Christopher \\ Moraes $^{2}$, Sidney Omelon ${ }^{4}$, Mark Driscoll ${ }^{1}$, Lawrence R. Chen ${ }^{3}$ \\ ${ }^{1}$ Department of Mechanical Engineering, ${ }^{2}$ Department of Chemical Engineering, ${ }^{3}$ Department of Electrical and \\ Computer Engineering, ${ }^{4}$ Department of Mining and Materials Engineering, McGill University, Montréal, Canada \\ lawrence.chen@mcgill.ca
}

\begin{abstract}
Reflective writing or exam wrappers appear to be a valuable exercise that may benefit students. This notion was evaluated in parallel within five engineering classrooms of different size and subject matter. The improvement or quantifiable impact of the reflective writing exercise differed from class to class. Despite this, overall results suggest that exam wrappers lend themselves well to improving learning, both for the students and instructors in the context of engineering classrooms.
\end{abstract}

Keywords: Exam Wrapper, Student Reflection.

\section{INTRODUCTION}

Self-regulated learning is the act of strategically and intentionally adapting learning activities to achieve learning goals [1-3]. Students can be encouraged to selfregulate their learning through reflective writing exercises which allows them to reconsider study techniques, exam preparation, and "soft skills" [4]. However, whether these exercises can improve understanding of technical, conceptual and design issues in Engineering remains unclear. Here, we investigated the effects of including a reflective writing component on student learning in a variety of engineering courses across four departments, with the objective of identifying situations in which such strategies are most effective.

We implemented exam wrappers, a structured reflective writing exercise [5], as a participation-based and integrated component of multiple assessments in five engineering courses that differed in level as well as type. Participating courses included the fundamental courses: Introduction to Signals and Systems (first-year course in Electrical and Computer Engineering), Fluid Mechanics (second-year course in Chemical Engineering), Machine Element Design (design-based third-year course in Mechanical Engineering), and two technical complimentary courses for final year/graduate students: Photonic Devices and Systems/Applications (Electrical and Computer Engineering), and Sustainable Materials Processing (Materials Engineering). Assessments included closedended problem solving, open-ended design, and technical writing skills. Classes ranged in size from 25 to 78 students. For each assessment, students completed 5 exam wrapper questions designed to prompt self-reflective learning either electronically or by hand. Anonymous surveys were conducted at the end of the course to determine how students viewed the experience.

Exam wrapper quality was evaluated using normalized rubrics that identified whether students produced a coherent, relevant, and thoughtful reflection. Grades from questions in subsequent assessments that were similar to those from previous assessments (quizzes, midterms, assignments) were tracked on an anonymized per-student basis, and correlated with quality of reflective writing. Grades on the exam wrappers were plotted vs. student grades, as well as the grade differences over multiple assessments.

A survey was given to the students, either during the semester (Electrical and Computer, and Chemical Engineering), or after the semester (Mechanical and Materials). The questions surveyed students for the impact of the exam wrapper exercise on their learning and study habits.

\section{EXAM WRAPPER METHODS}

The self-reflection exercise consisted of issuing exam wrapper question to students, evaluating the quality of the exam wrapper responses, tracking the student grades before and after an evaluation, and then correlating the change in student grades with the reflection response quality. Although there was variation in the specific implementation of the student self-reflection between departments, the change in academic performance with self-reflection quality and survey results will be presented for all departments.

\subsection{Exam Wrapper Questions and Quality Evaluation}

Examples of the most detailed Exam Wrapper questions include:

1. Which question(s) presented the greatest challenge? Why do you think that is?

2. After reviewing your assessment, why do you think you lost the most marks: Lack of 
understanding of the concept, Unsure what the question was asking, Careless or calculation errors, Not being able to apply concept in new contexts

3. Do the questions that presented the greatest challenge resemble previous practise examples (e.g., from an assignment)?

4. What is different between the assessment question and the one from previous examples?

5. What changes to your study habits do you plan to make when preparing for the next assessment?

The goals of these questions are for the student to reflect on the new course concept, reflect on factors that affected their performance, and consider how to improve future understanding and performance.

Each course used the Exam Wrapper strategy in different contexts. They will be briefly described here, as well the method of determining the reflection quality. The metrics used to determine the effect of the students' selfreflection on their performance are also described.

\subsection{Exam Wrapper Quality Evaluation and Tracking Student Performance Response}

Exam wrapper quality was evaluated using normalized rubrics that identified whether students produced a coherent, relevant, and thoughtful reflection.

The quality of the reflective writing exercises was scored according to a set of rubrics used for all courses. The rubric examined whether the students produced a coherent, relevant, and thoughtful reflection. Specifically, an ideal reflection was defined as one in which the student 'clearly summarizes a thorough and honest effort of selfassessment relative to pre-defined learning objectives, technical concepts, or evaluation questions.' Moreover, the reflection is 'complete, focused and detailed, and identifies clearly problematic matters and specifies a strategy that can be adopted to address weaknesses in understanding or exam preparation.' The reflections were scored on a scale of 0 to 3 , depending on the extent to which the reflection satisfied the following statement, 'the student's reflection aligns well with the description of an ideal reflection':

0: $\quad$ Strongly disagree

1: Disagree

2: $\quad$ Agree

3: $\quad$ Strongly agree

The rubrics and scoring system were designed to align with the four-category scheme developed by Kember et al.: (1) non-reflection (score of 0 ), (2) understanding (score of 1 ), (3) reflection (score of 2), and (4) critical reflection (score of 3 ).

Grades from questions in subsequent assessments that were similar to those from previous assessments (quizzes, midterms, assignments) were tracked on an anonymized per-student basis, and correlated with quality of reflective writing. Grades on the exam wrappers were plotted vs. student grades, as well as the grade differences over multiple assessments. Detailed execution methods for the four courses are described in Sections 2.2.1-2.2.4.

2.2.1. Introduction to Signals and Systems. This fundamental core course is taken by electrical and computer engineering students. It introduces students to notions of continuous time (CT) and discrete time (DT) signals and systems, their representations in the time, spectral, and transform domains, and the connection between CT and DT domains via sampling.

The course was taught using a flipped classroom approach where content was delivered outside of class via short videos and class time relied heavily on active learning strategies and techniques. Reflective writing was an instrumental part of the course and the exam wrapper is one exercise that was used to engage students in reflection following their midterm test. The graded tests were returned to the students in the class immediately following, i.e., within 72 hours. During this follow-up class, a quick overview of the test was provided and then students were asked to complete a questionnaire to guide their reflection and in particular, to identify gaps in their knowledge of concepts and technical content. Students were asked to complete or respond to the following

1. Go through the different questions and highlight directly on the test your error(s); explain your error(s) and/or any misunderstanding/misconception that led to the incorrect response(s). Do you now understand the source of your misunderstanding/misconception?

2. How did you prepare for this test?

3. How confident were you about your knowledge of the material before taking the test? And after taking the test?

4. Which question in the test was most challenging for you? Why do you think that is?

5. Based on your response to the question above, does this question resemble a question previously covered, e.g., in a class activity or on an assignment? What is different between this test question and the previous question(s)?

6. What changes do you need to make to prepare for the next test? Is this realistic?

Completing the exam wrapper was worth 2 points out of 40 of the midterm grade.

The exam wrapper was scored by the instructor as well as a graduate research assistant who also served as a TA for 
the course. The scores were then added to obtain a total score out of 6 .

2.2.2. Machine Element Design. The course with 41 enrolled students held 2 exams: a midterm covering static and fatigue failure, shafts, and keys design; and a final covering gears, springs, bearings, screws, and fasteners design. Exam-wrappers were utilized after the midterm electronically via Socrative, an open-source application designed for effective engagement and on-the-fly assessments.

The exam-wrapper used was composed of 4 fundamental questions relating to the objectives mentioned earlier:

1. Based on how demanding you thought the course is, and how long you spent preparing for this midterm, what would be your response to the following sentence: I am satisfied with my performance and grade received (strongly disagree/disagree/neutral/agree/strongly agree).

2. What type of questions on the midterm presented the most challenge to you? And why do you think that?

3. After looking over your midterm, why do you think you lost the most points? (Lack of conceptual understanding/question was unclear/careless or calculation mistake/unable to apply concepts in new contexts).

4. What changes to your study habits do you plan to make when preparing for the next midterm?

Data were collected and analyzed using statistical analyses in Excel. To quantify and normalize the exam-wrappers, each was a given a score between 1 and 10, with 10 being the highest. Trying to eliminate any subjectivity, the scoring criteria was as follows: The student starts with a 10 and then:

1. If the answer to any of the two close-ended questions do not agree with the answer to any of the two open-ended ones, the student loses 1 point for each. (Incoherence)

2. If the student scored below average, yet is satisfied with his/her grade, he/she loses 2 point. (Discouragement)

3. If the student did not identify appropriate changes to the study habits based on the answer to question 3,3 points are lost. (lack of self-regulation and goal adjustment)

The last two points are lost if the reflection shows any lack of relevancy, consistency, or objectivity.

The metric to assess students' performance and improvement was defined as the grade difference between the two taken exams. This was then plotted against the reflection quality.
2.2.3. Fluid Mechanics. This course provides students with an introduction to hydrostatics, control volumes, mass and momentum conservation, dimensional analysis and boundary layers. 76 students were enrolled in this course. Students were given seven days after receiving their graded quizzes and exams to submit their reflection and a scanned copy of their exam. The Exam Wrapper was worth 1 point out of 10 for each of the two quizzes and 1 point out of 30 for the midterm. The exam wrappers were scored by the course teaching assistant.

2.2.4. Sustainable Materials Design. Students were given the option to submit a reflection on their performance in a detailed technical outline. They were asked to reflect on the low performances as described in a detailed grading rubric. Students were given a $10 \%$ bonus if they submitted the self-reflection with the resubmission. The course instructor graded the resubmission, and a research assistant evaluated the reflection quality on a scale of 10 , which was binned to 3 groups for comparison with other course results. The percentage change in grade before and after the resubmission was the performance response.

\subsection{Survey Questions}

The on-line survey queried five questions of the students in all courses:

1. the number of minutes spent completing the reflective writing exercise $(<1 \mathrm{~min} /<5 \mathrm{~min} /<10$ $\min />10 \min$

2. how often they referred to their reflective writing (never/once/a few times/often),

3. if they tried the reflective writing exercise in other courses (strongly disagree/disagree/neither agree nor disagree/agree/strongly agree),

4. if they used the reflective writing as a tool when preparing for class or assessments (strongly disagree/disagree/neither agree nor disagree/agree/strongly agree),

5. if, overall, the reflective writing exercise contributed to their learning (not a chance/a few times/a lot).

This voluntary survey was issued digitally to the students.

\section{CORRELATION OF REFLECTION QUALITY WITH CHANGE IN STUDENT PEFORMANCE}

The change in student performance as a function of the quality of the self-reflection is described for each department in Sections 3.1-3.4. 


\subsection{Introduction to Signals and Systems}

A detailed description of this study will also be presented in these proceedings [6], which follow previous and related studies [7, 8]. Figure 1 illustrates that students with a higher quality of reflection on the EW also tended to perform better on the final for the type of question that posed them greater difficulty (i.e., the grades on Q1F and Q3F were higher compared to Q1M and Q3M).

(a)

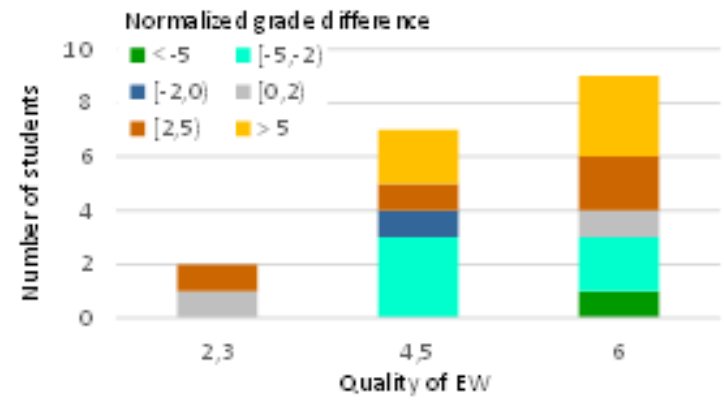

(b)

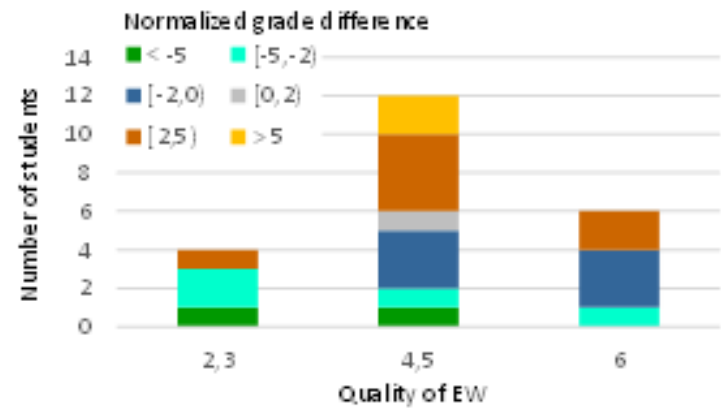

Figure 1: Normalized grade difference (histogram coloured section) presented with respect to the number of students with a given grade difference (ordinate) versus the exam wrapper quality (abscissa)

\subsection{Machine Element Design}

Looking at the quality of reflection written by the students after doing the midterm, no specific correlation was observed (Fig. 2). However, in general, it seemed that most students who scored a relatively low grade, between $65 \%$ and $78 \%$, put efforts to reflect on the exam, and ended up with a reflection score between 6 and 8 out of 10. On the other hand, some of those who did well on the midterm, scored between $80 \%$ and $93 \%$ submitted brief reflections that were given low scores, between 3 and 5 out of 10 . Figure 2 also shows that other outliers existed where 2 students scored low grades $(52.5 \%$ and $71.5 \%)$, but also reflected poorly (1 and 2 reflection quality out of 10 ). Similarly, 4 students who performed well on their midterm (87.5\%-95\%), scored a very high reflection quality (9 and 10 out of 10$)$.

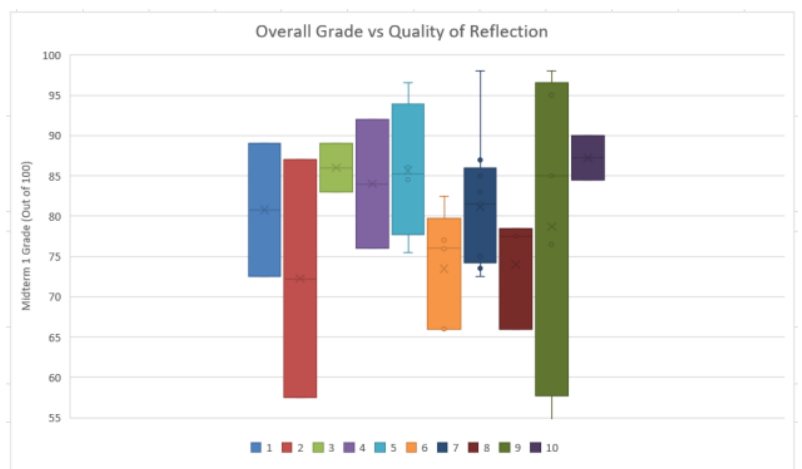

Figure 2: Midterm 1 grades in relation to reflection quality.

In considering the objective of whether students were able to self-assess their performance, this was only observed with students who scored a low grade. Analyzing their answers to the question of whether they were satisfied or not with their performance, most of those students responded as highly unsatisfied, which was intuitive. However, when the exam-wrappers directed them at identifying the mistakes, some amazing responses were collected such as: "I think I focused more on the theory part and forgot to solve as many exercises as possible", "It was a fair exam but I should go back and review statics, which is where most of my mistakes came from", ...etc. That is, when students were asked to identify specific mistakes, and were motivated to objectively answer exam-wrappers questions, they tended to spend time and effort going over their exam again, and were actually able to spot specific mistakes they did. Therefore, most students, especially those who did not do well on their midterm, were able to self-assess themselves objectively.

Furthermore, to investigate the impact of examwrappers, the second midterm grades, undertaken after submitting the midterm-wrappers, were plotted against the reflection quality (Fig. 3). A general increasing trend was observed where, students who submitted a relatively better reflection, scored a relatively high grade in the second midterm.

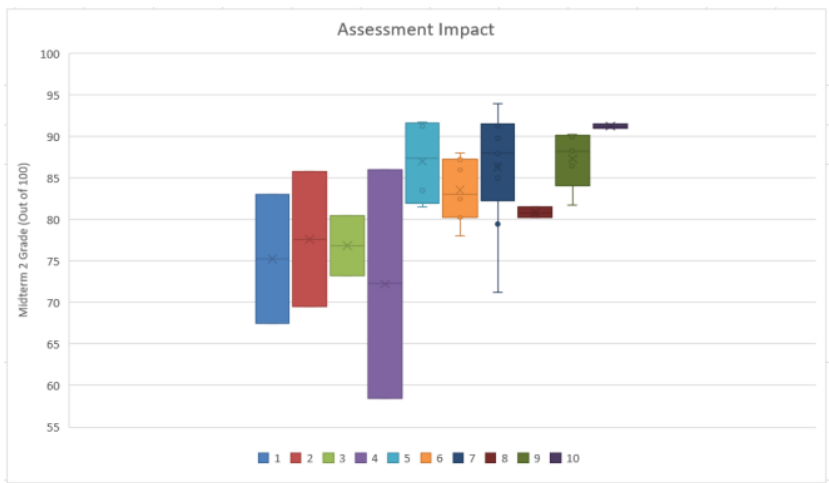

Figure 3: Midterm 2 grades in relation to reflection quality.

In considering another objective of the usefulness of exam-wrappers and whether such a tool has an impact or 
not, a trend proving its potential impact was observed (Fig. $3)$. After the fluctuations observed in the case of the first midterm, results were more normalized when comparing the reflection quality to the second midterm. When looking at some responses to that matter, students were able to identify the following mistakes: "careless or calculation mistakes", "not being able to apply concepts in new contexts", etc. Other more detailed responses and how students would adjust their study behaviors were: "better organizing my notes to spare time during the exam", "spend more time on theory in preparation for the short answers", "work in groups and solve practice problems with my friends", ...etc.

What further proved the usefulness and impact of examwrappers was the other brief comments submitted by students who initially scored good grades in their first midterm. Such answers ranged from "not much" to "none", although being have received a grade around $85 \%$. Because those students identified their grades as satisfactory, their behavior was attributed to the fact that they felt that their studying strategy is perfect and that they will be able to achieve similar good scores in the second exam by following the same studying strategies. Conversely, especially those two students in particular, their grade dropped to $71.5 \%$ and $73.25 \%$ in the second midterm.

Finally, to get the full picture of how the reflections resulted in a change in students' grades, improvement in grades between the first and second midterms were plotted against the quality of reflections (Fig. 4). Students with low reflection quality (8 students with a quality of 4 at most) show fluctuations, that is, only one student improved by 12.5 points while the rest showed score deterioration, with one dropping by 17.5 points. Impressively, as the reflection quality improved, student grades improved, with the highest being a 47.5 increase in grade for a student who scored a 10/10 reflection (Fig. 4).

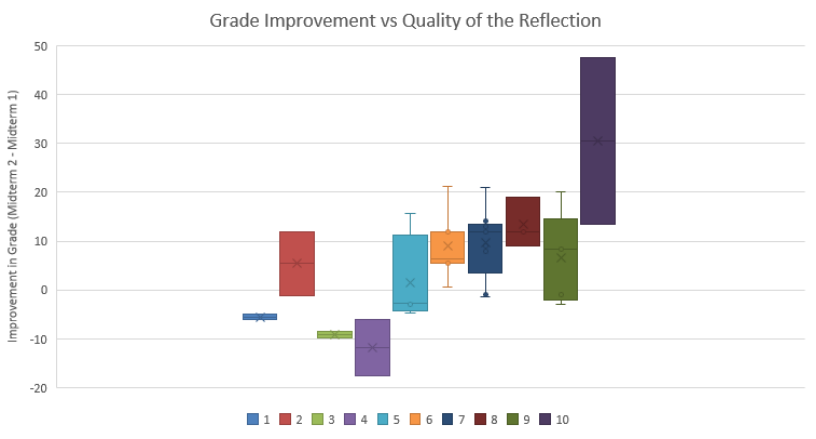

Figure 4: Grade improvement in relation to the reflection quality.

\subsection{Fluid Mechanics}

Students completed reflections on the Fluid Mechanics quiz and midterm questions that they deemed most difficult. Many of the quiz/midterm problems were similar in concept to the problems in the final exam. We, therefore, sought to evaluate the relationship between the quality of these reflections and the score improvement between the quizzes/midterm and final exam. There was a large variability in students' grade improvements between evaluations (Fig. 5 a-c), and, as a result, there was no significant trend between quality of reflection and student grades.
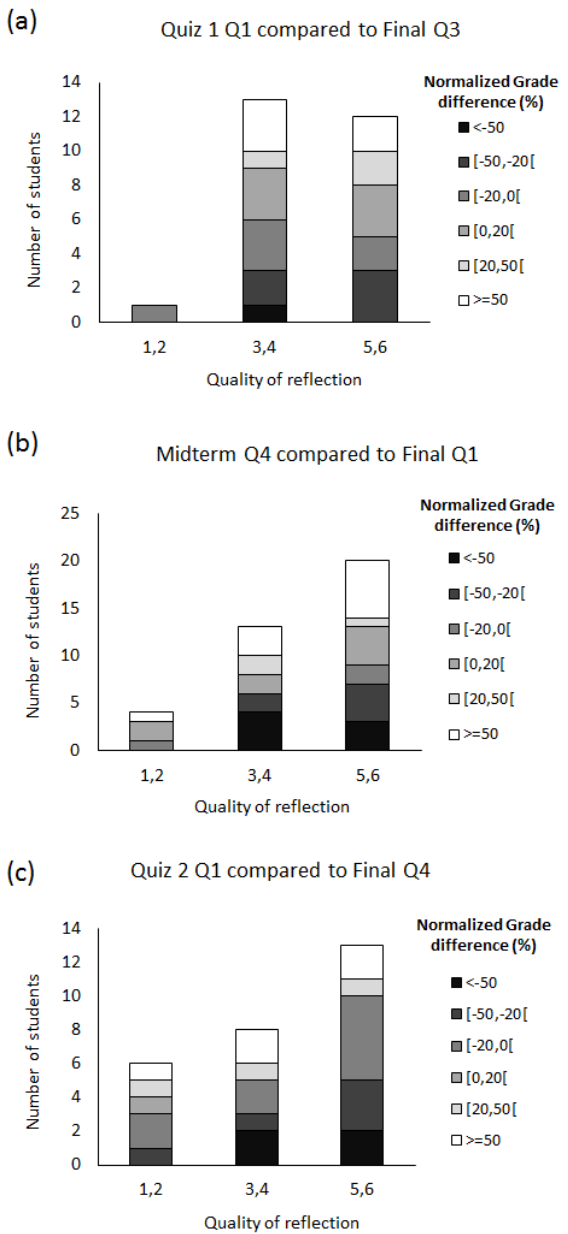

Figure 5: Fluid mechanics normalized grade improvement between (a) Quiz 1 question 1 and final exam question 3, (b) Midterm question 4 and final question 1, (c) Quiz 2 question 1 and final question 4. Grades were normalized based on the question class average to correct for differences in difficulty between quiz/midterm questions and final exam questions.

The implementation of an exam reflection in the Fluid Mechanics course therefore did not show an enhancement of student performance.

\subsection{Sustainable Materials Design}

The reflection quality was assessed by a teaching assistant who was not associated with the course. A boxplot 
of the reflection quality versus the change in resubmission grade is presented in Fig. 6.

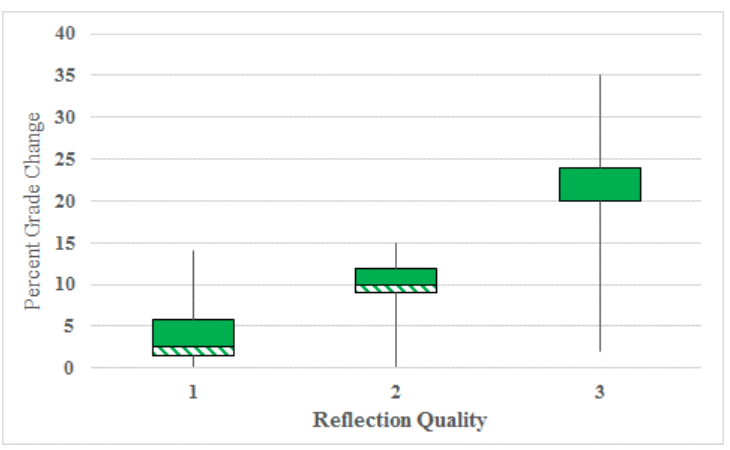

Figure 6: Boxplot of the percent grade improvement, binned according to a low $(1, n=4)$, medium $(2, n=5)$, or high $(3, n=9)$ reflection quality.

A positive relationship between the improvement of student grade over an initial submission and the quality of their reflection was observed.

\section{SURVEY RESULTS}

Figure 7 shows the results of the voluntary student survey that was given to students in four of the courses. The data is presented as the percent of students who responded to the survey. All students spent more than one minute on their reflection exercise. The largest percentage of students that responded with the longest time spent writing their reflection were in the Introduction to Signals and Systems course. These students did not spend less than 5 minutes on their reflection exercise.

In general, students did not refer often to their reflection. $15-40 \%$ of the student replied that they referred to their reflection a few times, once, or never. Students reported that they agreed or neither agreed or disagreed that they used their reflective writing as a tool when preparing for class or assignments.

Minutes spent doing the reflective writing exercise.

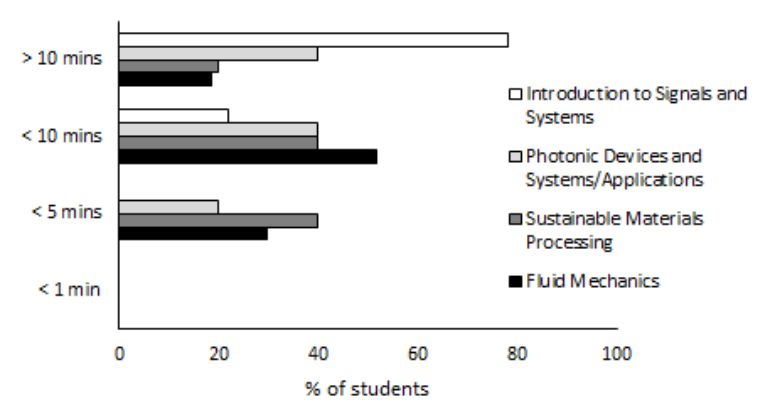

I have referred to my reflective writing...

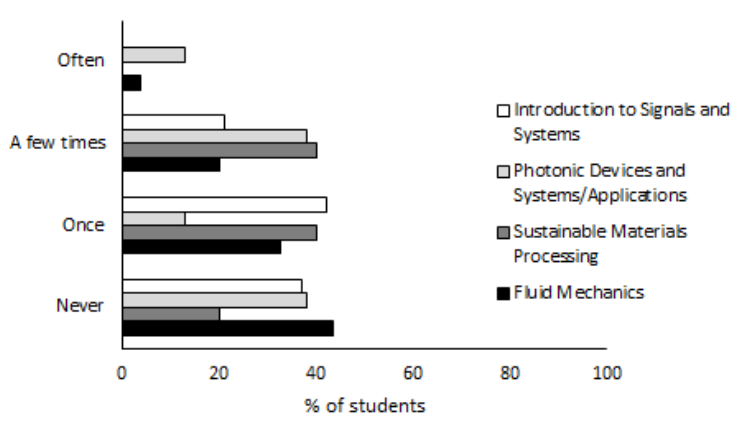

I used my reflective writing as a tool when preparing for class or assessments.

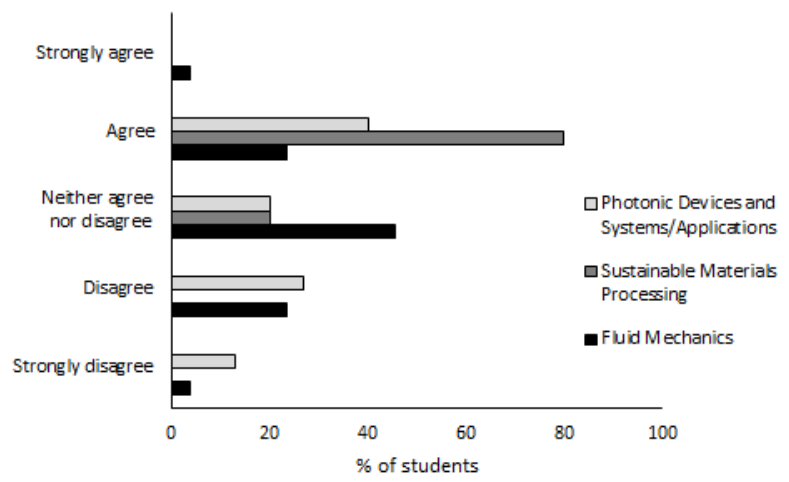

Overall, the reflective writing exercises contributed to my learning.

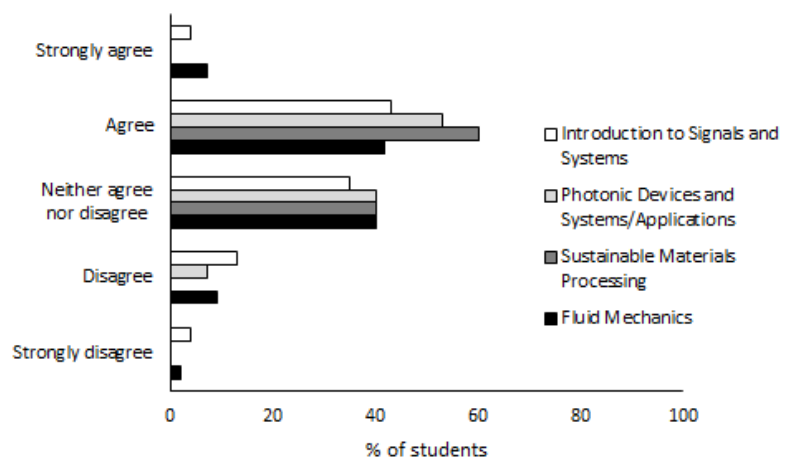

I have tried reflective writing in other courses.

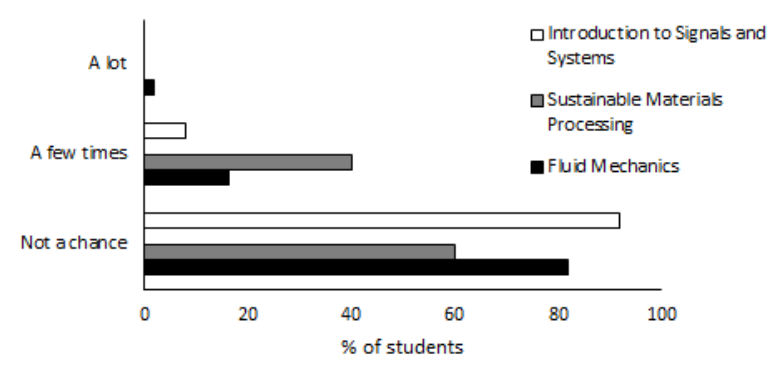

Figure 7: Survey results from students in Introduction to Signals and Systems, Photonic Devices and Systems/Applications, Sustainable Materials Processing and Fluid Mechanics. 
When queried about the contribution of the reflection exercise to the quality of their learning, the highest percentage of respondents for all courses agreed that the reflection contributed to their learning (41-60\%). The next most popular response was that students neither agreed nor disagreed that the reflection contributed to their learning (35-40\%). Less than $15 \%$ strongly agreed, disagreed, or strongly disagreed that this exercise contributed to their learning. Most respondents for all courses surveyed (60-90 $\%)$ reported that they did not try reflective writing in their other courses. Between 10 and $40 \%$ replied that they tried it a few times. Less than $5 \%$ of the fluid mechanics students reported to have used this reflection technique "a lot" in other courses.

\section{DISCUSSION}

Quality of reflection remained reasonably consistent even over multiple uses within the same course. Improvements in student grades varied greatly between courses, perhaps due to the inherent differences in study techniques used in fundamental vs design courses. In design courses, high quality reflections more strongly correlated with grade improvement than in foundational classes. Additionally, variability of grade improvement may also be affected by differences in delivery of the exam wrapper exercise between instructors. For example, in Signals and Systems and Sustainable Materials Processing, students reworked questions and handed in the corrected assessment with their reflections. These students exhibited a more pronounced grade improvement when compared to Fluid Mechanics students who were only required to produce written reflections. Students did not refer to their reflections very often, but it is possible that once the error or cognition problem was resolved, reviewing the reflection was not considered to be necessary.

\section{CONCLUSION}

When surveyed at the end of the semester, students in all five courses believed that reflective writing contributed to their learning. While there may be many reasons for this perception amongst students, exam wrappers were certainly useful for instructors to identify concrete problems, which may not be achieved with other strategies. These findings suggest that exam wrappers can positively influence learning and pedagogy across engineering courses, but the greatest gains can be achieved only by careful design and delivery of the exercise.

\section{Acknowledgements}

We appreciate and acknowledge the funding that allowed for this study by the McGill University eLATE Grassroots
Initiative. The authors acknowledge the support and efforts of the graduate students who undertook the reflection grading, including Rana Yekani.

\section{References}

[1] Linda B. Nilson, Creating self-regulated learners: Strategies to strengthen students' self-awareness and learning skills. Sterling, VA: Stylus Publishing, 2013. \{ISBN: 978-1579228675\}

[2] Barry J. Zimmerman, "Theories of self-regulated learning and academic achievement: An overview and analysis," in Self-regulated learning and academic achievement, Barry J. Zimmerman and Dale H. Schunk (eds.) New York, NY: Routledge, 2013. \{eBook ISBN: 9781135659141\}

[3] Philip H. Winne and Allyson F. Hadwin, "Selfregulated learning and socio-cognitive theory," in International Encyclopedia of Education, $3^{\text {rd }}$ ed. Penelope Peterson, Eva Baker and Barry McGaw (eds.) Elsevier, 2010. \{ISBN: 978-0-08-044894-7\}

[4] Saundra Yancy McGuire, Teach Students How to Learn: Strategies You Can Incorporate into any Couse to Improve Student Metacognition, Study Skills, and Motivation. Stylus Publishing LLC, 2015, 288 pp. \{ISBN-13: 978-1620363164\}

[5] Marsha C. Lovett, "Make Exams Worth More than the Grade: Using Exam Wrappers to Promote Metacognition", in Using Reflection and Metacognition to Improve Student Learning: Across the Disciplines, Across the Academy, Matthew Kaplan, Naomi Silver, Danille LaVaque-Manty, and Deborah Meizlish. $1^{\text {st }}$ ed., Sterling, VA: Stylus Publishing LLC, 2013, p. 18-52 \{ISBN: 978-1-57922-827-9\}

[6] Lawrence R. Chen, Maxime Jacques, and Zeinab Sobhanigavgani, Proc. CEEA Canadian Engineering Education Conf., CEEA19 (Ottawa, ON; 8-12 June 2019), paper ID\#159.

[7] David Benson and Haolin Zhu, "Student reflection, self-assessment and categorization of errors on exam questions as a tool to guide self-repair and profile student strengths and weaknesses in a course," in Proc. $122^{\text {nd }}$ ASEE Annual Conference \& Exposition (Seattle, WA; 14-17 June 2015), paper ID\#12366.

[8] Renee M. Clark and Samuel J. Dickerson, “Assessing the impact of reflective activities in digital and analog electronics courses," IEEE Transactions on Education, in press, 2019. 